\title{
MEASUREMENT OF BEAM SIZE AT THE PHOTON FACTORY WITH THE SR INTERFEROMETER
}

\author{
M. Katoh and T. Mitsuhashi* \\ High Energy Accelerator Research Organization, Oho, Tsukuba, Ibaraki, 305-0801 Japan
}

\section{Abstract}

The beam size at the low emittance configuration of the Photon Factory was measured by the use of the SR interferometer. The spatial coherence of the visible SR beam was measured for both of the vertical and horizontal directions with the wavelengths at $500 \mathrm{~nm}$ and $633 \mathrm{~nm}$. From the measured spatial coherence of the SR beam, the beam size measurements of $87.3 \mu \mathrm{m}$ in the vertical and $261.2 \mu \mathrm{m}$ in the horizontal were obtained. The observed horizontal beam size agreed with the designed beam size.

\section{INTRODUCTION}

The SR interferometer, which was developed by one of the authors, has good sensitivity for small beams. Since this method is based on the spatial coherence of th ${ }^{*}$ SR beam, it is suitable for measuring small electron beam sizes[1],[2][3]. The principle of object-profile measurement by means of the spatial coherency of the light is known as van CitterutZernike's theorem[4]. In this time, we measured both the vertical and the horizontal beam sizes at the high brilliant configuration of the Photon Factory. In the measurement of interferogram as a function of spatial frequency by the use of SR interferometer, we ordinary use the method which is scanning the double slit separation. In other hand, we can measure the spatial coherence by using the method which is scanning the wavelength. Using these two method together, we can measure the spatial coherence in wider spatial frequency region. In this time we measured the spatial coherence with different two wavelengths for the check of using these two methods.

\section{SPATIAL COHERENCE AND BEAM SIZE}

According to van Citterut-Zernike's theorem, the profile of an object is given by the Fourier Transform of the complex degree of spatial coherence at longer wavelengths such as visible light. Let $\boldsymbol{f}$ denote the beam profile as a function of position $y_{0}$, and $\gamma$ denote the complex degree of spatial coherence as a function of spatial frequency $v$. Then $\gamma$ is given by the Fourier transform of $\boldsymbol{f}$ as

$$
\gamma(v)=\int f\left(y_{0}\right) \exp \left\{-i 2 \pi v \cdot y_{0}\right\} d y_{0}
$$

follows;

The interferogram which is observed with the SR interferometer (see Fig.1, below) $I\left(y_{1}\right)$ is given by,

\footnotetext{
*Email: mitsuhas@mail.kek.jp
}

$I\left(y_{1}\right)=I_{0}\left[\sin c\left(\frac{2 \pi a}{\lambda R_{1}} y_{1}\right)\right]^{2} \cdot\left[1+|\gamma(v)| \cos \left(\frac{2 \pi D}{\lambda R_{1}} y_{1}+\varphi\right)\right]$

where $a$ denotes the half of slit height of the double slit $D$, denotes the double slit separation, $R_{1}$ denotes distance between the interferogram and the back principle point of objective lens of the interferometer, and $\varphi$ denotes the phase of the interference fringe. The spatial frequency $v$ is given by

$$
v=\frac{2 \pi}{\lambda R_{0}} D
$$

where $R_{0}$ is distance between the object beam and the double slit. Two methods are available to measure the interferogram as a function of the spatial frequency, one is scanning double slit separation, and other is scanning the wavelength. Using equation (2), we can measure the degree of spatial coherence from the interferogram. Using equation (1), we can measure the beam profile by Fourier transform of the spatial coherence, and under the assumption of a Gaussian beam profile, we can measure the RMS beam size from the interferogram.

\section{SR INTERFEROMETER}

The SR interferometer is basically a wavefrontdivision-type two-beam interferometer using polarized quasi-monochromatic rays. A schematic drawing of the SR-interferometer is shown in Fig.1.

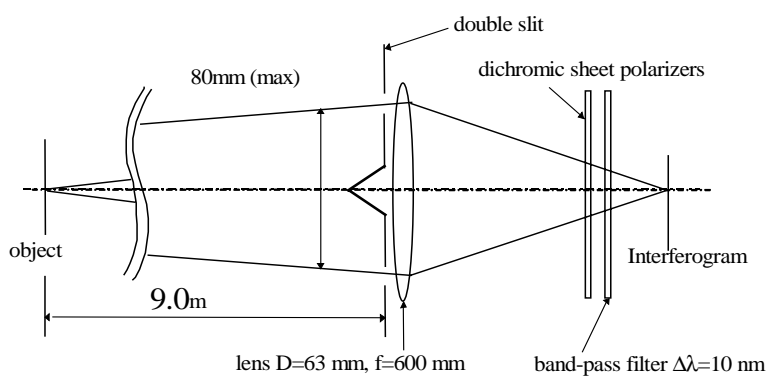

Fig.1 Outline of the SR interferometer.

The double slit assembly is located in the front of the objective lens as close as possible(few $\mathrm{mm}$ ). We use an aperture of 1 (width) $\times 2$ (height) $\mathrm{mm}^{2}$ for the double slit assembly. A diffraction limited doublet lens having a diameter of $63 \mathrm{~mm}$ and a focusing length of $f=600 \mathrm{~mm}$ was used as an objective. A dichroic sheet polarization filter ( extinction ratio: $10^{-4}$ ) and a band-pass filter of $10 \mathrm{~nm}$ bandwidth was used to obtain a polarized quasi-monochromatic ray. The distance between source point and double slits of the SR interferometer is $9.03 \mathrm{~m}$. 


\section{VERTICAL BEAM SIZE MEASUREMENT}

4-1. Measurement of the absolute value (visibility) of the complex degree of spatial coherence in the vertical direction.

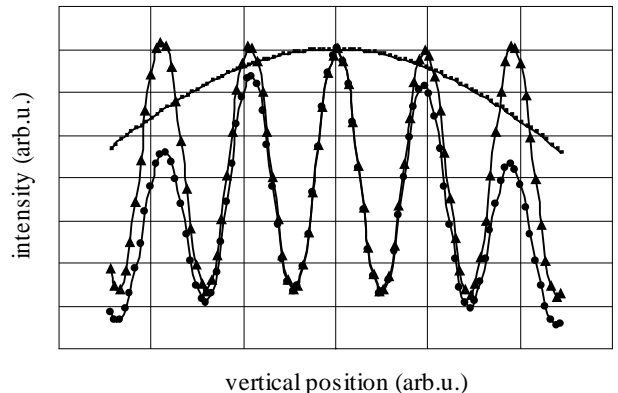

(a)

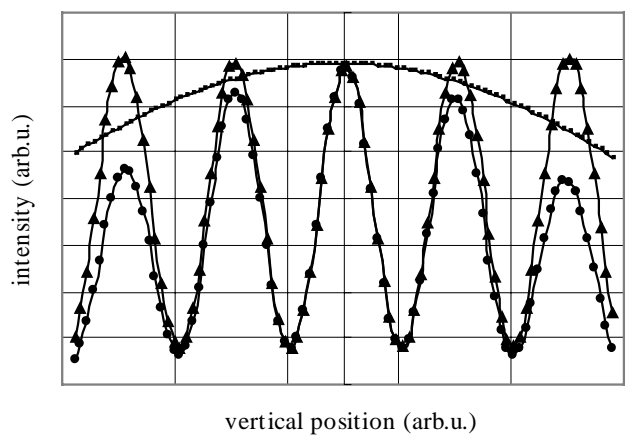

(b)

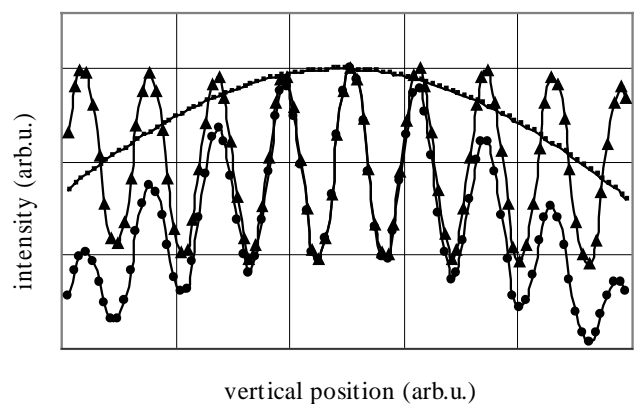

(c)

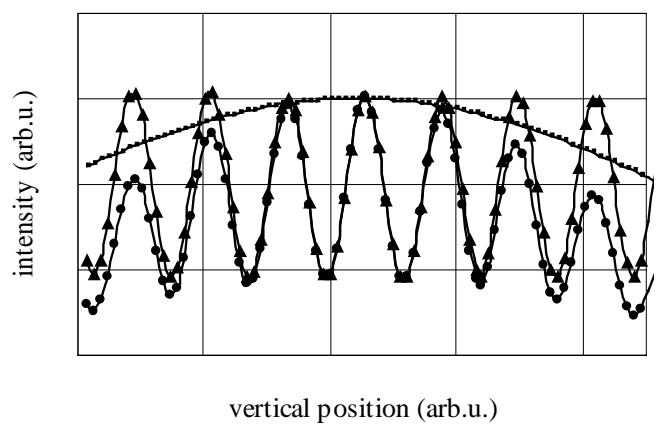

(d)

Fig. 2. Observed vertical interferograms. (a) $D=7 \mathrm{~mm}$, $\lambda=500 \mathrm{~nm}$, (b) $D=7 \mathrm{~mm}, \lambda=633 \mathrm{~nm}$, (c) $D=12 \mathrm{~mm}$, $\lambda=500 \mathrm{~nm}$, and (d) $D=12 \mathrm{~mm} \lambda=633 \mathrm{~nm}$.
The interferogram was measured by changing the double slit separations $D$ between the two slits from $3 \mathrm{~mm}$ to $22 \mathrm{~mm}$ in $1.0 \mathrm{~mm}$ steps at the two wavelengths of 500nm and 633nm. Four examples of observed interferograms are shown in Fig.2. From Fig.2, we can see that the contrast of the interference fringe is reduced when the double-slit separation is increased, due to the finite size of the beam.

\section{4-2. Evaluation of vertical beam size from spatial} coherence measurement.

The absolute value of the complex degree of spatial coherence is evaluated from the visibility of the observed interferogram. The results are shown in Fig. 3.

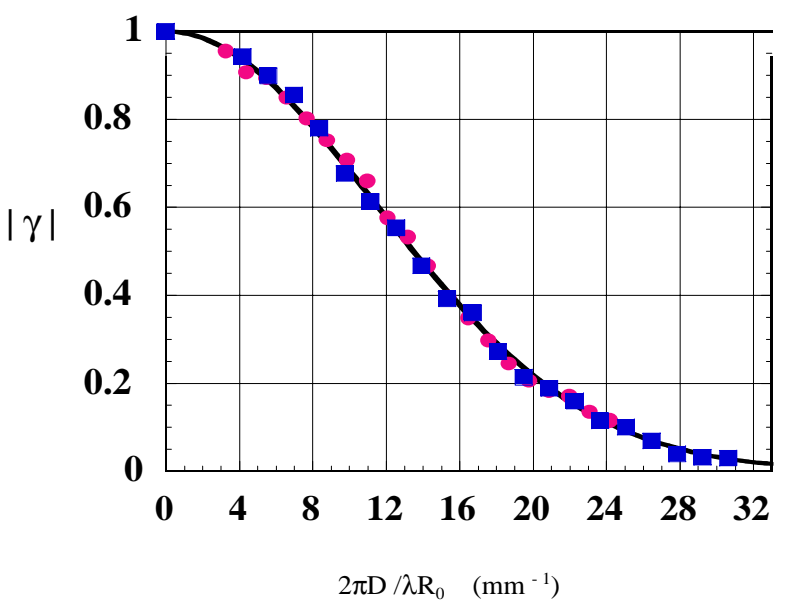

Fig.3. Absolute value of the complex degree of spatial coherence in the vertical direction. The round spots denotes the spatial coherence measured with $\lambda=633 \mathrm{~nm}$, and the triangular spots denotes the spatial coherence measured with $\lambda=500 \mathrm{~nm}$

From Fig.3, two results of the spatial coherence measured at wavelengths of $500 \mathrm{~nm}$ and $633 \mathrm{~nm}$ are agreed each other. Least-squares fitting of the spatial coherence by using a Gaussian beam profile is also shown in Fig.3. Since the result of fitting seems very good, we can conclude that the approximation of Gaussian profile to the beam profile is good enough. We can evaluate the RMS beam size from this fitting, and the result of the beam size is $87.3 \pm 0.4 \mu \mathrm{m}$.

\section{5 . HORIZONTAL BEAM SIZE MEASUREMENT}

5-1. Measurement of the absolute value (visibility) of complex degree of spatial coherence in the horizontal direction

The absolute value of the complex degree of spatial coherence for horizontal direction was measured in the same way as in the vertical direction, but with the double-slit rotated by $90^{\circ}$. Four examples of observed interferograms are shown inFig.4. 


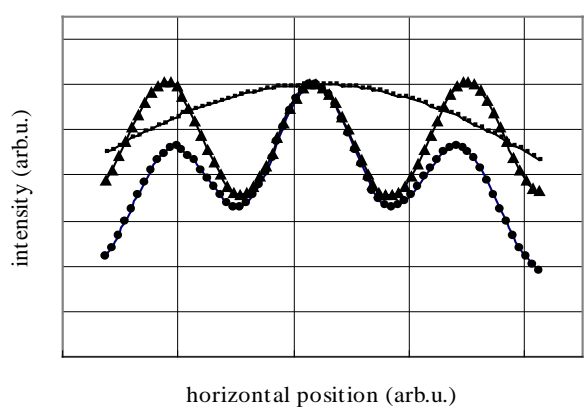

(a)

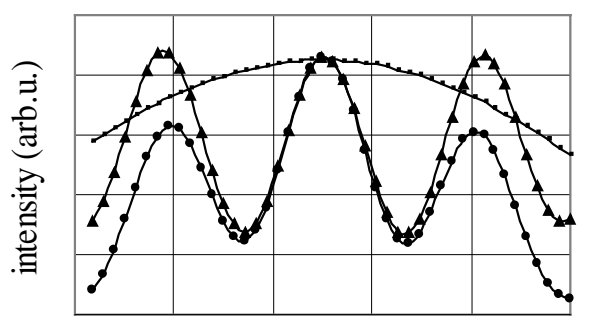

horizontal position (arb.u)

(b)

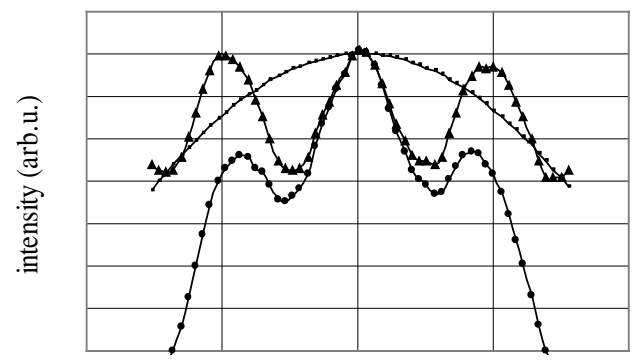

horizontal position (arb.u.)

(c)

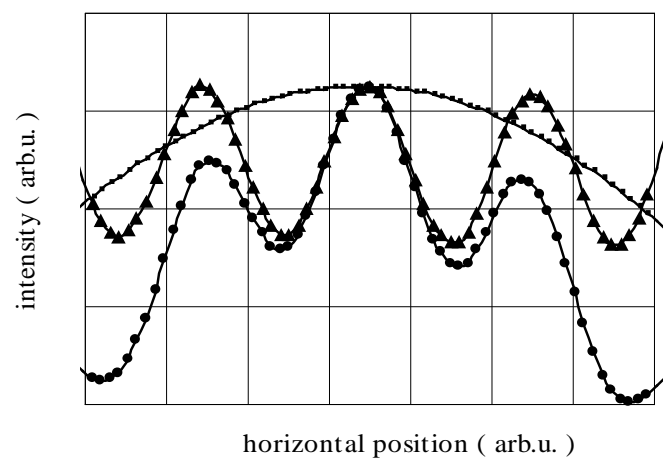

(d)

Fig. 4. Observed horizontal interferograms. (a) $D=5 \mathrm{~mm}, \lambda=500 \mathrm{~nm}$, (b) $D=5 \mathrm{~mm}, \lambda=633 \mathrm{~nm}$, (c) $D=7 \mathrm{~mm}, \lambda=500 \mathrm{~nm}$, and (d) $D=7 \mathrm{~mm}, \lambda=633 \mathrm{~nm}$.

\section{5-2.Result of Degree of Spatial Coherence and Horizontal Beam Size}

The absolute value of the complex degree of coherence is evaluated from the visibility of the measured interferogram. The results are shown in Fig. 5.

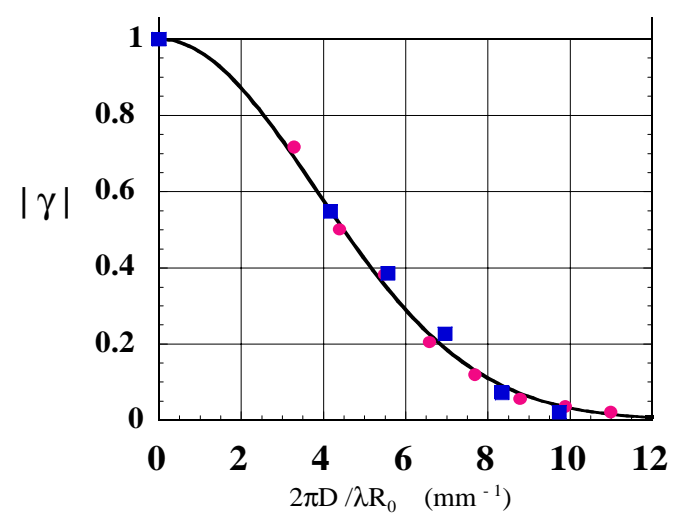

Fig.5. Absolute value of the complex degree of spatial coherence in the horizontal direction. The round spots denotes the spatial coherence measured with $\lambda=633 \mathrm{~nm}$, and the triangular spots denotes the spatial coherence measured with $\lambda=500 \mathrm{~nm}$

From Fig.5, two results of the spatial coherence measured at wavelengths of $500 \mathrm{~nm}$ and $633 \mathrm{~nm}$ are agreed each other. Least-squares fitting of the spatial coherence by using a gaussian beam profile including field depth effect is also shown in Fig.3. We can evaluate the RMS beam size from this fitting, and the result of the beam size is $261.2 \pm 2.6 \mu \mathrm{m}$.

\section{CONCLUSIONS}

The vertical and horizontal beam sizes of the low emittance configuration of the Photon Factory were measured via SR interferometer. The two results of the spatial coherence measured at wavelengths of $500 \mathrm{~nm}$ and $633 \mathrm{~nm}$ are agreed each other. We conclude the vertical beam size is $87.3 \mu \mathrm{m}$ and the horizontal beam size is $261.2 \mu \mathrm{m}$. The designed beam sizes are $263 \mu \mathrm{m}$ (using design values of energy spread and measured $\beta$ and $\eta$-functions) in the horizontal and $110 \mu \mathrm{m}$ in the vertical ( $2 \%$ coupling is assumed). The observed beam size in the horizontal direction agreed with the estimated beam size. We achieved designed emittance in horizontal, and $1.26 \%$ coupling.

\section{ACKNOWLEDGMENTS}

The authors wish to thank to Mr. M. Shimoda for his help on data analysis. The authors also thank the Photon Factory operation group for their support.

\section{REFERENCES}

[1] T. Mitsuhashi, Proc of 1997 Particle Accelerator Conference, 766,(1997), Vancouver.

[2] T. Mitsuhashi, H. Iwasaki, Y. Yamamoto, T. Nakayama and D.Amano, Proc. 11th Symposium on Accelerator Science and Teqchnology 441 (1997).

[3] T. Mitsuhashi and T. Naitoh, Proc.of 6th European Particle Accelerator Conference 1565,(1998), Stockholm.

[4]M. Born and E. Wolf,"Principles of Optics, P459, Pergamon press. (1980). 